\title{
Выбор условий для безреагентного (необменного) разделения аминокислотсодержащих смесей на анионообменниках
}

\author{
(C) 2019 Антропова Е.М., Трунаева Е.С., Каширцева Е.P., \\ Хохлова О.Н., Хохлов В.Ю.
}

ФГБОУ ВО «Воронежский государственньий университет», Воронеж

Поступила в редакцию 15.11.2019 г.

DOI: $10.17308 /$ sorpchrom.2019.19/2233

Работа посвящена исследованию влияния высоты и диаметра слоя сорбента, плотности его упаковки, характеристик гидродинамических режимов, а так же концентрации и соотношения компонентов раствора на вид выходных кривых при сорбционно-десорбционных процессах.

Установлено, что разделение аминокислотсодержащих смесей необходимо проводить на низком и широком слое мелкодисперсного сорбента в солевой форме при отсутствии свободного объема в верхней и нижней части колонки с использованием низких скоростей подачи раствора, чередуя стадии сорбции и десорбции. Процесс повторяется несколько циклов для полного разделения компонентов, при этом для десорбции используется вода. Для исследуемой системы на стадии сорбции триптофан переходит в сорбент, и раствор обедняется по аминокислоте, а концентрация соли остается неизменной. Таким образом, уже на первой стадии десорбции получают чистый раствор этой аминокислоты. Десорбция поглощенного компонента эффективно проводится водой при использовании количеств, сопоставимых с объемом раствора с разделяемой смесью.

Ключевые слова: необменная сорбция, аминокислота, анионообменник.

\section{Conditions for reagent-free (non-exchange) separation of amino acids containing mixtures on anion exchangers}

\author{
(C) 2019 Antropova E.M., Trunaeva E.S., Kashirtseva E.R., \\ Khokhlova O.N., Khokhlov V.Yu. \\ Voronezh state University, Voronezh
}

Since in non-exchange sorption the development along the column is accompanied by a significant blurring due to molecular diffusion and leads to a significant decrease in the efficiency of separation in comparison with ion exchange, the aim of the work is establish rational conditions for the separation of amino acid-containing mixtures by ion exchangers at non-exchange absorption conditions.

The work is devoted to the study of the influence of the sorbent layer height, diameter, the density of its packaging, the characteristics of hydrodynamic regimes, as well as the concentration and ratio of the solution components on the appearance of output curves in sorption-desorption processes.

Since the main condition of reagentless separation amino acids's mixtures is what use of ion exchangers in salt form, which eliminates overcharging and securing zwitterion ion exchange, demineralization of amino acid solutions was studied on the mixture of tryptophan and calcium chloride with different content of highly basic components at high based anion exchanger AV-17-2P in Cl-form.

It was found that the separation of amino acid-containing mixtures should be carried out under the follow conditions: the low and wide layer of fine sorbent in salt form in the absence of free volume in the 
upper and lower parts of the column; low rates of solution supply; alternating stages of sorption and desorption. The process is repeated several cycles to completely separate the components with using of water for desorption.

For studying system tryptophan passes into the sorbent and the solution is depleted in amino acid the salt concentration remains unchanged at the sorption stage. Thus, at the first stage of desorption, a pure solution of this amino acid is obtained. Desorption of the absorbed component is effectively carried out by water using quantities comparable to the volume of the solution with the separated mixture.

Keywords: non-exchange sorption, amino acid, anion exchanger

\section{Введение}

Поскольку при необменном поглощении силы взаимодействия сорбата с сорбентом слабые $[1,2]$, то десорбировать поглощенное вещество можно водой. Это положено в основу безреагентного метода разделения веществ. Процесс проводят в две стадии: сорбцию осуществляют в условиях необменного поглощения, при этом поглощается компонент с большей сорбционной способностью, и раствор обедняется по данному веществу; десорбцию проводят водой, при этом из сорбента вымывается поглощенный компонент. При необходимости цикл повторяют неоднократно. При этом сорбент остается в исходной ионной форме, поэтому не требуется использование дополнительных реагентов для регенерации ионообменника, что уменьшает экологические и экономические проблемы по сравнению с ионным обменом. Метод впервые предложен для разделения кислот и их солей на анионите, содержащем тот же анион, что и в разделяемой смеси, и назван Acid Retardation - «удерживания кислоты» [3], развит и применен в ряде других работ [4-7].

Для эффективного разделения веществ на ионообменниках необходимо подобрать условия проведения сорбционно-десорбционных процессов. При этом надо учитывать, что при необменной сорбции движение фронта по колонке сопровождается существенным размытием за счет молекулярной диффузии и приводит к значительному снижению эффективности разделения веществ по сравнению с ионным обменом [8]. Потому целью работы являлось установление рациональных условий разделения аминокислотсодержащих смесей на ионообменниках в условиях необменного поглощения.

\section{Эксперимент}

Основным условием безреагентного разделения аминокислотсодержащих смесей является использование ионообменников в солевой форме, что исключит перезарядку и ионообменное закрепление цвиттерлитов.

Разделение органо-минеральных смесей, в которых органическим компонентом является аминокислота, с различной эффективностью возможно как на высоко-, так и низкоосновных сорбентах [9-11]. Однако использование последних характеризуется меньшим количеством поглощенного вещества на стадии сорбции и гидролизом ионной формы ионита на стадии десорбции водой. Поэтому для деминерализации растворов аминокислот использовали высокоосновный анионообменник AB-17-2П в Cl-форме. Деминерализацию растворов аминокислот исследовали на примере смеси триптофана с хлоридом кальция с различным содержанием компонентов. Состав исходного раствора обеспечивает отсутствие анионов в растворе, способных к ионному обмену с исследуемым анионитом - используемая соль не гидролизуется, что определяет наличие нейтральной среды в растворе, аминокислота в растворе находится в виде биполярного иона, перезарядка и ионообменное закреп- 
ление в фазе сорбента не возможно, поэтому протекает необменное поглощение обоих компонентов.

Для уменьшения размывания фронта сорбции необходима минимизация свободного объема колонки (в верхней и нижней части, межгранульного пространства), подача раствора снизу для гравитационного подавления процесса, подбор скорости подачи раствора и др. $[4,8]$, поэтому в работе исследовано влияние высоты и диаметра слоя сорбента, плотности его упаковки, характеристик гидродинамических режимов, а так же концентрации и соотношения компонентов раствора на вид выходных кривых при сорбционно-десорбционных процессах.

На рис.1 представлена принципиальная схема безреагентного разделения аминокислотсодержащих смесей.

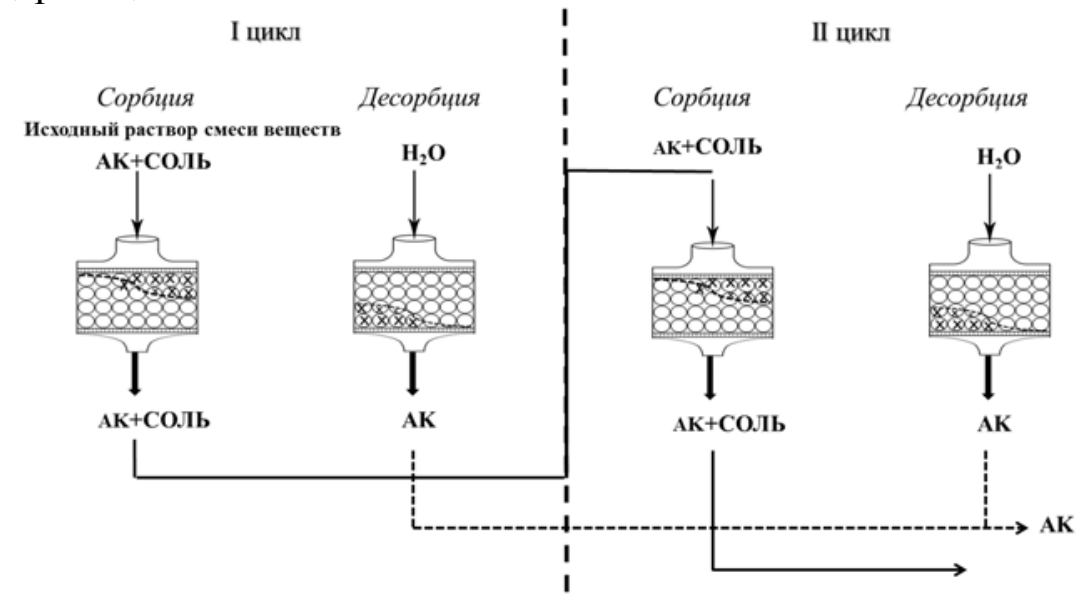

Рис. 1. Схема безреагентного разделения смеси аминокислоты с минеральной солью.

В верхнюю часть колонки помещался пористый поршень, который обеспечивал наличие плотного слоя сорбента и равномерность подачи раствора по всему сечению колонки. Нижняя часть колонки представляла собой плоскость, обеспечивающую отсутствие свободного объема и равномерный сток жидкости. Объем очищаемого раствора $200 \mathrm{~cm}^{3}$, объем собираемых фракций $v=10 \mathrm{~cm}^{3}$.

\section{Обсуждение результатов}

Для повышения эффективности разделения аминокислотных и органоминеральных смесей исследовано влияние высоты слоя сорбента на форму фронта сорбции на примере смеси триптофан-хлорид кальция с концентрацией компонентов 0.05 моль/дм ${ }^{3}$ и соотношением 1:1 на анионообменнике AB-17-2П в Cl-форме. Приблизительно одинаковый объем набухшего сорбента $\left(\mathrm{V}=16 \pm 0.6 \mathrm{~cm}^{3}\right)$ помещали в колонки различного диаметра, получая необходимую высоту слоя анионообменника АВ-17-2П (Cl): (1) d=3.7 см, h=1.5 см, (2) d=1.9 см, h=6 см, (3) d=1.5 см, h=9 см. В исследуемой сорбционной системе соль не сорбируется и полностью остается в растворе, а аминокислота переходит в анионообменник согласно своим сорбционным способностям [12]. Поэтому на выходе из колонки концентрация $\mathrm{CaCl}_{2}$ соответствовала исходной, а триптофана становится меньше. Таким образом, собираемый раствор обеднялся по аминокислоте.

Зависимость вида фронта сорбции от высоты слоя сорбента представлена на рисунке 2. Как видно из рисунка, начальный участок выходных кривых сорбции триптофана практически совпадает, а конечный существенно размывается, что свидетельствует о внутридиффузионном лимитировании процесса $[13,14]$. При увели- 
чении высоты слоя сорбента наблюдается усиление размытия конечного участка и сорбционного фронта в целом. С одной стороны, при этом увеличивается количество поглощенного триптофана, с другой стороны, размытие фронта сорбции не выгодно при практическом использовании процесса. Исходя из полученного, исследование влияния остальных условий сорбции вели на низком и широком слое сорбента (рис.2-6), что соответствует литературным данным о практическом использовании подобных процессов $[3,13]$.

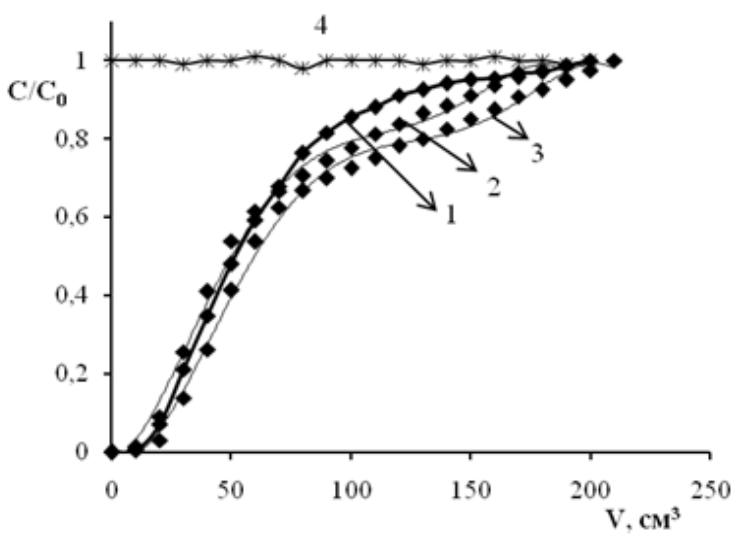

Рис. 2. Зависимость вида выходной кривой триптофана (1-3) и $\mathrm{CaCl}_{2}$ (4) от высоты слоя сорбента в системе $\mathrm{AB}-17-2 \Pi(\mathrm{Cl})+\mathrm{Trp}+\mathrm{CaCl}_{2}$ (0.05:0.05моль/дм $\left.{ }^{3}\right), \mathrm{d}=3.7 \mathrm{~cm}, \mathrm{~h}=1.5 \mathrm{~cm}$ (1), $\mathrm{d}=1.9 \mathrm{~cm}, \mathrm{~h}=6 \mathrm{~cm}(2), \mathrm{d}=1.5 \mathrm{~cm}$, $\mathrm{h}=9 \mathrm{~cm}(3)$.

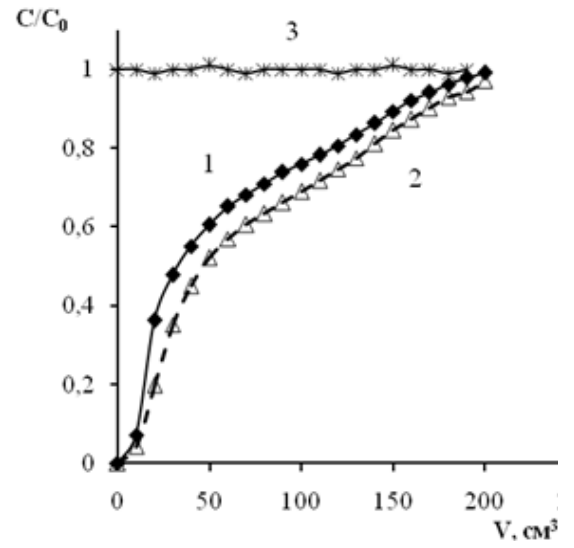

Рис. 3. Зависимость вида выходной кривой триптофана $(1,2)$ и $\mathrm{CaCl}_{2}(3)$ от диаметра зерна сорбента в системе $\mathrm{AB}-17-2 \Pi(\mathrm{Cl})+\mathrm{Trp}+\mathrm{CaCl}_{2}$ (0.05:0.05 моль/дм $\left.{ }^{3}\right)$, диаметр зерна 0.250.5 мм (1) и более 0.5 мм (2), (условия $v=1.8 \mathrm{~cm}^{3} /$ мин, $\left.\mathrm{d}=3.7 \mathrm{~cm}, \mathrm{~h}=1.5 \mathrm{~cm}\right)$

Для уменьшения размытия сорбционного фронта при движении по колонке необходимо уменьшить количество межгранульного раствора за счет уплотнения слоя сорбента при использовании зерен меньшего диаметра. Исследованы фракции ионообменника $\mathrm{AB}-17-2 П(\mathrm{Cl})$ с диаметром 0.25-0.5 мм и более 0.5 мм. Получено, что свободный объем колонки при использовании мелкой фракции составляет $15 \%$, а при использовании крупной - 30\%. Таким образом, использование мелкой фракции сорбента обеспечивает меньшее количество межгранульного раствора.

На рисунке 3 представлены выходные кривые сорбции смеси $\operatorname{Trp}+\mathrm{CaCl}_{2}$ 0.05:0.05 моль/дм ${ }^{3}$ на анионообменнике различного фракционного состава (высота слоя $1.5 \mathrm{~cm}$, скорость подачи раствора $1.8 \mathrm{~cm}^{3} /$ мин). Из рисунка видно, что использование мелкодисперсного сорбента обеспечивает меньшее размывание фронта сорбции за счет молекулярной диффузии.

Использованный для дальнейшего исследования сорбент содержит преимущественно мелкодисперсные зерна анионообменника ( $80 \%)$, что обеспечивает выполнение вышеизложенных условий. Исследовано влияние скорости подачи раствора на размывание фронта сорбции. На рисунке 4 представлены выходные кривые сорбции смеси триптофан - хлорид кальция при скоростях пропускания раствора $1.8 \mathrm{~cm}^{3} /$ мин и $3.0 \mathrm{~cm}^{3} /$ мин (высота слоя $1.5 \mathrm{~cm}$, сорбент содержит $80 \%$ зерен с диаметром 0.25-0.5 мм, концентрации компонентов 0.05 моль/дм $\left.{ }^{3}\right)$.

Из рисунка видно, что при высокой скорости подачи раствора в растворе на выходе из колонки содержится больше триптофана, чем при низких скоростях процесса, т.е. сорбция ухудшается из-за того, что не успевает устанавливаться сорбционное равновесие. Таким образом, использование высоких скоростей подачи раство- 
ра не целесообразно, поэтому дальнейшее разделение вели при скорости подачи раствора $1.8 \mathrm{~cm}^{3} / \mathrm{Mин}$.

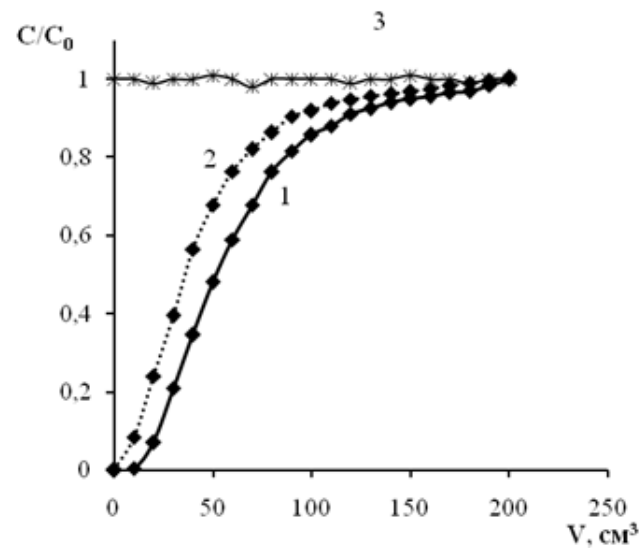

Рис. 4. Зависимость вида выходной кривой триптофана $(1,2)$ и $\mathrm{CaCl}_{2}(3)$ от скорости подачи раствора в системе $\mathrm{AB}-17-2 П(\mathrm{Cl})+\operatorname{Trp}+\mathrm{CaCl}_{2}\left(0.05: 0.05 \mathrm{моль} /\right.$ дм $\left.^{3}\right)$, $v=1.8 \mathrm{~cm}^{3} /$ мин, $\mathrm{d}=3.2 \mathrm{~cm}, \mathrm{~h}=1.5 \mathrm{~cm}(1) ; \nu=3 \mathrm{~cm}^{3} /$ мин, $\mathrm{d}=3.2 \mathrm{~cm}, \mathrm{~h}=1.5 \mathrm{~cm}(2)$.

Исследовано разделение смеси триптофан-хлорид кальция при различных соотношениях компонентов - 0.025:0.05 моль/дм ${ }^{3}$, 0.05:0.05 моль/дм³ ${ }^{3}$, 0.075:0.05 моль/дм ${ }^{3}$. Установлено, что независимо от соотношения аминокислоты и соли в растворе вид выходных кривых остается неизменным. Во всех случаях триптофан поглощается ионообменником согласно своим сорбционным способностям - при увеличении концентрации раствора увеличивается количество поглощенной аминокислоты, а соль остается в растворе.

Таким образом, выявлены условия для предотвращения размывания фронта сорбции и наиболее эффективного разделения веществ в условиях необменного по-

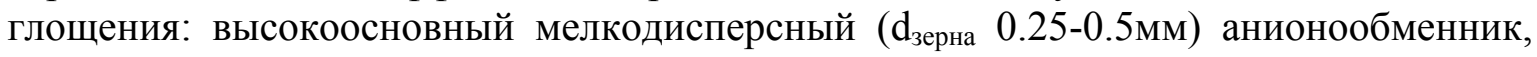
имеющий тот же противоион, что и удаляемый электролит, низкий и широкий слой сорбента $(\mathrm{d}=3.7 \mathrm{~cm}, \mathrm{~h}=1.5 \mathrm{~cm})$, малая скорость пропускания раствора $\left(1.8 \mathrm{~cm}^{3} /\right.$ мин $)$ при любом соотношении компонентов. Подача раствора снизу для подавления размытия фронта сорбции под действием силы тяжести не изменяет характеристики сорбционных кривых, но технически усложняет процесс.

Использование рациональных условий проведения сорбции-десорбции приводит к существенному изменению характеристик разделения веществ даже с близкими характеристиками сорбции - фенилаланина и триптофана (рис. 5).

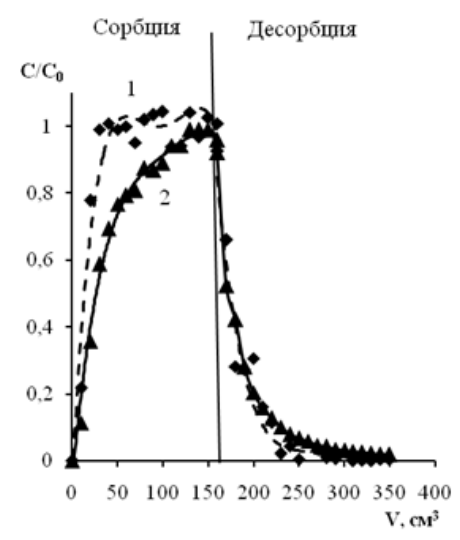

(a)

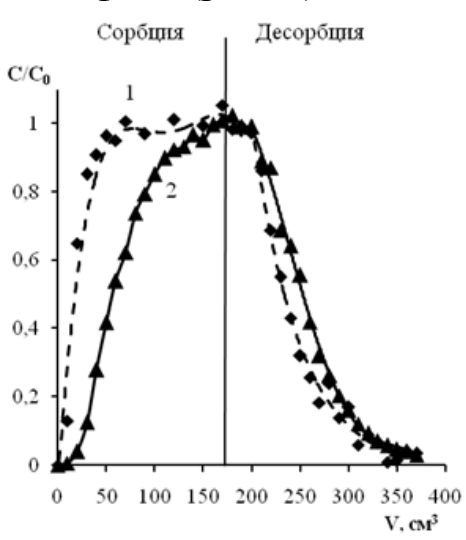

(б)

Рис. 5. Выходные кривые $\mathrm{Phe}^{ \pm}(1)+\operatorname{Trp}^{ \pm}(2)$ на AB-17-2П(Cl) в произвольных условия $\mathrm{h}=11 \mathrm{~cm}, \mathrm{~d}=1 \mathrm{~cm}, \mathrm{v}=2 \mathrm{~cm}^{3} /$ мин (а) и в подобранных условиях $\mathrm{h}=1.5 \mathrm{~cm}$, $\mathrm{d}=3.7 \mathrm{~cm}, \mathrm{v}=2 \mathrm{~cm}^{3} /$ мин (б). 
Для разделения смеси триптофан-хлорид кальция с содержанием компонентов $0, .5$ моль/дм ${ }^{3}$ каждого на анионообменнике AB-17-2П в Cl-форме использовалось циклическое повторение процессов сорбции-десорбции (водой). На стадии сорбции триптофан переходит в сорбент, и раствор обедняется по аминокислоте, а концентрация соли остается неизменной. На стадии десорбции водой из ионообменника вымывается поглощенный триптофан. Установлено, что 95-99\% поглощенного вещества вымывается $200 \mathrm{~cm}^{3}$ воды, а для полного удаления триптофана из анионообменника необходимо $250 \mathrm{~cm}^{3}$ десорбента. Таким образом, уже на первой стадии десорбции получают чистый раствор этой аминокислоты (рис. 6).

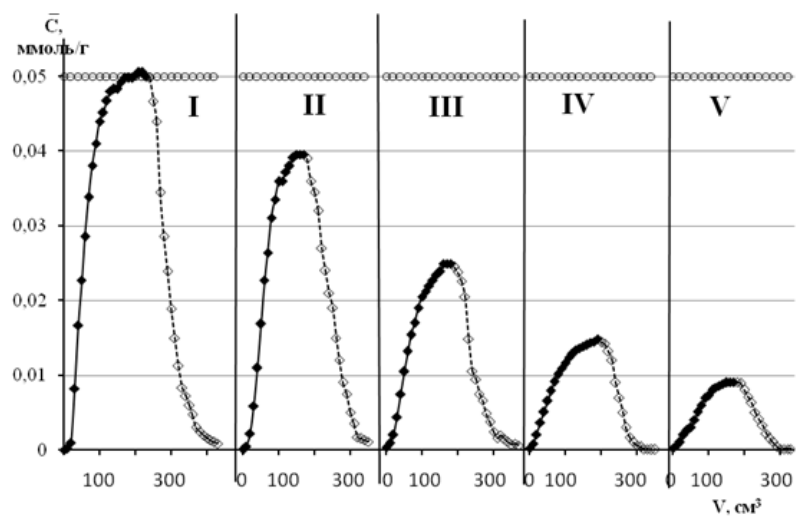

Рис. 6. Вид концентрационных профилей триптофана

(-จ- сорбция, - $\diamond$ - десорбция) и хлорида кальция (-О-) при циклическом повторении процесса разделения при смеси $\operatorname{Trp}^{ \pm}+\mathrm{CaCl}_{2}\left(0.05: 0.05\right.$ моль/дм $\left.{ }^{3}\right)$ на $\mathrm{AB}-17-2 П(\mathrm{Cl})$

$$
\left(v=1.8 \mathrm{~cm}^{3} / \text { мин, } \mathrm{d}=3.7 \mathrm{~cm}, \mathrm{~h}=1.5 \mathrm{~cm}\right) \text {. }
$$

Раствор, собранный в процессе сорбции пропускают через ту же колонку, так как ионообменник остается в исходной ионной форме $\left(\mathrm{Cl}^{-}\right)$. Процесс повторяется до требуемой степени очистки компонентов (рис.1, рис. 6). Видно, что за пять циклов можно удалить триптофан из смеси на 93\%.

\section{Заключение}

Таким образом, разделение аминокислотсодержащих смесей необходимо проводить на низком и широком слое мелкодисперсного сорбента в солевой форме при низких скоростях подачи раствора. При этом десорбция поглощенного компонента эффективно проводится водой при использовании количеств, сопоставимых с объемом раствора с разделяемой смесью. Процесс повторяют циклически для достижения необходимой степени очистки аминокислоты.

\section{Список литературы}

1. Трунаева Е.С, Хохлова О.Н., Хохлов В.Ю. // Сорбиионные и хроматографические проиессы. 2018. Т. 18. № 2. С. 142-149.

2. Трунаева Е.С, Хохлова О.Н., Хохлов В.Ю. // Журнал структурной химии. 2017. Т. 58. № 1. C. 23-28.

3. Hatch M.J, Dillon J.A. // I\&EC Process Design and Development. 1963. Vol. 2. No 4. pp. 253-263.
4. Хамизов Р.Х. // Сорбционные и хроматографические проиессы. 2013. Т. 13. № 5. C. 600-604.

5. Крачак А.Н., Хамизов Р.Х., Познухова В.А. и др. // Сорбиионные и хроматографические прочессы. 2011. Т. 11. № 1. С. 77-88.

6. Крачак А.Н., Хамизов Р.Х., Долгоносов А.М. и др. // Сорбичонные и хромато- 
графические прочессы. 2014. Т. 14. № 6. С. 902-911.

7. Ferapontov N.B. et al. // Reactive and Functional Polymers. 2000. Vol. 45. pp. 145153.

8. Ферапонтов Н.Б. Дис. д-ра хим. наук. M. 2001. 298 c.

9. Трунаева Е.С, Хохлова О.Н. // Журнал структурной химии. 2013. Т. 13. Вып. 5. С. 655-662.

10.Карлашова Т.С., Трунаева Е.С., Хохлова О.Н., Хохлов В.Ю. // Сорбиионные и хроматографические проиессы. 2014. Т. 14. № 4. С. 648-653.

\section{References}

1. Trunaeva E.S, Khokhlova O.N., Khokhlov V.Yu., Sorbtsionnye $i$ khromatograficheskie protsessy, 2018, Vol.18, No 2, pp. 142-149.

2. Trunaeva E.S, Khokhlova O.N., Khokhlov V.Yu., Zhurnal strukturnoi khimii, 2017, Vol. 58, No 1, pp. 23-28.

3. Hatch M.J, Dillon J.A., I\&EC Process Design and Development, 1963, Vol. 2, No 4, pp.253-263.

4. Khamizov R.Kh., Sorbtsionnye i khromatograficheskie protsessy, 2013, Vol. 13, No 5, pp. 600-604.

5. Krachak A.N., Khamizov R.Kh., Poznukhova V.A. et al., Sorbtsionnye i khromatograficheskie protsessy, 2011, Vol. 11, No 1, pp. 7788.

6. Krachak A.N., Khamizov R.Kh., Dolgonosov A.M. et al., Sorbtsionnye i khromatograficheskie protsessy, 2014, Vol. 14, No 6, pp. 902911.

7. Ferapontov N.B. et al., Reactive and Functional Polymers, 2000, Vol. 45, pp. 145-153.

Антропова Елена Михайловна - магистр 2-го года обучения кафедры аналитической химии, Воронежский Государственный Университет, Воронеж.

Трунаева Евгения Сергеевна - к.х.н., кафедра аналитической химии, Воронежский Государственный Университет, Воронеж.

Каширцева Елизавета Романовна - магистр 2-го года обучения кафедры аналитической химии, Воронежский Государственный Университет, Воронеж.

Хохлова Оксана Николаевна - доцент кафедры аналитической химии, к.х.н., Воронежский Государственный Университет, Воронеж.

Хохлов Владимир Юрьевич - профессор кафедры аналитической химии, д.х.н., Воронежский Государственный Университет, Воронеж.
11.Кожухова Е.Ю., Трунаева Е.С., Хохлова О.Н. // Сорбиионные и хроматографические прочессы. 2015. Т. 15. № 5. С. 657-663.

12.Хохлова О.Н. // Вестник Воронежского государственного университета. 2015. № 2. C. $42-45$.

13.Гельферих Ф. Иониты: Основы ионного обмена. М. Изд-во Иностран. литер. 1962. $490 \mathrm{c}$.

14.Кокотов Ю.А., Пасечник В.А. Равновесие и кинетика ионного обмена. Л. Наука. 1977. $336 \mathrm{c}$.

8. Ferapontov N.B. Dis. d-ra chem. nauk. Moscow, 2001, $298 \mathrm{p}$.

9. Trunaeva E.S, Khokhlova O.N., Zhurnal strukturnoi khimii, 2013, Vol. 13, No 5, pp. 655-662.

10.Karlashova T.S., Trunaeva E.S., Khokhlova O.N., Khokhlov V.Yu., Sorbtsionnye i khromatograficheskie protsessy, 2014, Vol. 14, No 4, pp. 648-653.

11.Kozhukhova E.Yu., Trunaeva E.S., Khokhlova O.N., Sorbtsionnye $i$ khromatograficheskie protsessy, 2015, Vol. 15, No 5, pp. 657663.

12.Khokhlova O.N., Vestnik Voronezhskogo gosudarstvennogo universiteta, 2015, No 2, pp. 42-45.

13. Gelferih F. Ionity: Osnovyionnogoobmena, M., Izd-vo Inostran. liter., 1962, 490 p.

14.Kokotov Yu.A., Pasechnik V.A. Ravnovesie i kinetika ionnogo obmena, L., Nauka Publ., $1977,336 \mathrm{p}$.

Antropova Elena M. - master 2nd year of study, department of analytical chemistry, Voronezh State University, Voronezh, e-mail: antropovaalena1@yandex.ru

Trunaeva Eugenia S. - associate professor, department of analytical chemistry, Voronezh State University, Voronezh, e-mail: evgeniya.tru@bk.ru

Kashirtseva Elizaveta R. - master 2nd year of study, department of analytical chemistry, Voronezh State University, Voronezh e-mail: kashirtseva_e@mail.ru

Khokhlova Oksana N. - associate professor, department of analytical chemistry, Voronezh State University, Voronezh, e-mail: okxox@yandex.ru

Khokhlov Vladimir Yu. - professor, department of analytical chemistry, Voronezh State University, Voronezh, e-mail: vladkh70@mail.ru 